\title{
Construction and Measurement of the Pre-Series Twin Aperture Resistive Quadrupole Magnet for the LHC Beam Cleaning Insertions
}

\author{
G. de Rijk, S. Bidon, E. Boter, G. S. Clark, O. Hans, M. Racine, and A. Salinas
}

\begin{abstract}
CERN's Large Hadron Collider (LHC) requires 48 twin aperture resistive quadrupoles in the beam cleaning insertions. Canada is contributing these magnets to CERN in the framework of the TRIUMF-LHC collaboration contracts. A pre-series magnet was produced by Canadian industry and delivered in March 2001. This magnet incorporates important design changes that resulted from experience with a prototype magnet. The construction of this pre-series magnet and the measurements made at ALSTOM and at CERN are reported. A comparison is made between high precision pole distance measurements and the magnetic measurements performed with a rotating coil mole. Conclusions for series production and possibilities for multipole corrections are outlined.
\end{abstract}

Index Terms-Accelerator magnet, quadrupole, resistive.

\section{Cleaning Insertions}

$\mathbf{T}$ HE LHC beams are collimated in the IR3 and IR7 beam cleaning insertions. In these insertions only resistive magnets can be used, due to the shower particles originating from the collimators [1]. The twin aperture resistive quadrupoles installed in the cleaning insertions are called the MQW magnets.

\section{PROJeCt History}

The prototype magnet was designed in 1996 and delivered in 1998 by ALSTOM Canada [2]. Following mechanical and magnetic measurements, a series of design modifications were made. The pre-series magnet, MQW001, was built to this modified design. In March 2001 it was delivered to CERN for evaluation. Series production has now begun. The last magnet is scheduled to be delivered in December 2003.

\section{DESIGN MODIFICATIONS}

A cross section of the pre-series magnet is shown in Fig. 1. The magnet's main parameters are listed in Table I. A photograph of the magnet is shown in Fig. 2.

The magnet core contains six stacks of laminations of two types, as shown in Fig 1. The centrally mounted Type1 stacks have two poles. The laterally mounted Type 2 stacks have one

Manuscript received September 24, 2001. This work was supported by the National Research Council of Canada.

G. de Rijk, S. Bidon, E. Boter, and O. Hans are with CERN, Geneva, Switzerland.

M. Racine and A. Salinas are with ALSTOM Canada Inc., Tracy, Quebec, Canada.

G. S. Clark is with TRIUMF, Vancouver, B.C., Canada.

Publisher Item Identifier S 1051-8223(02)03429-2.

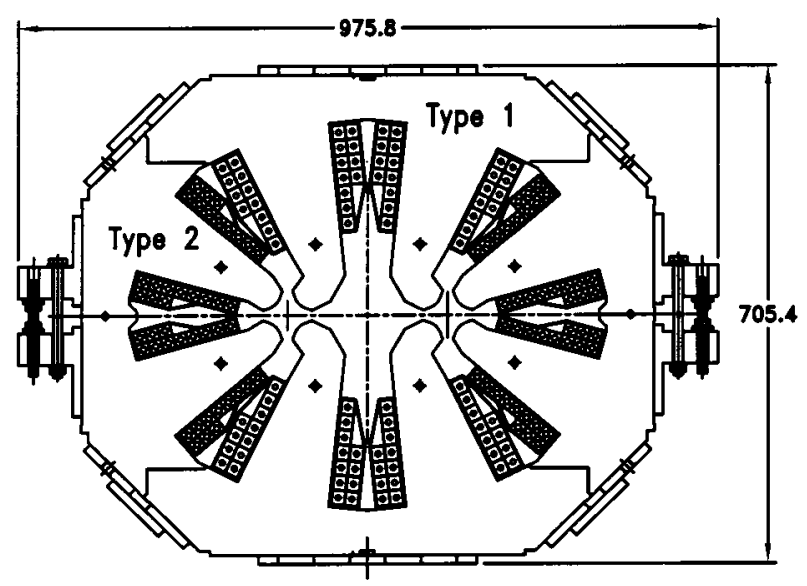

Fig. 1. Cross section of the pre-series magnet MQW001.

TABLE I MQW MAIN PARAMETERS

\begin{tabular}{ll}
\hline \hline Aperture diameter & $46 \mathrm{~mm}$ \\
\hline Aperture centre distance & $224 \mathrm{~mm}$ \\
\hline Nominal gradient & $35 \mathrm{~T} / \mathrm{m}$ \\
\hline Nominal current & $710 \mathrm{~A}$ \\
\hline Yoke length & $3100 \mathrm{~mm}$ \\
\hline Overall length & $3400 \mathrm{~mm}$ \\
\hline Yoke width & $975.8 \mathrm{~mm}$ \\
\hline Yoke height & $705.4 \mathrm{~mm}$ \\
\hline Total weight & $11500 \mathrm{~kg}$ \\
\hline \hline
\end{tabular}

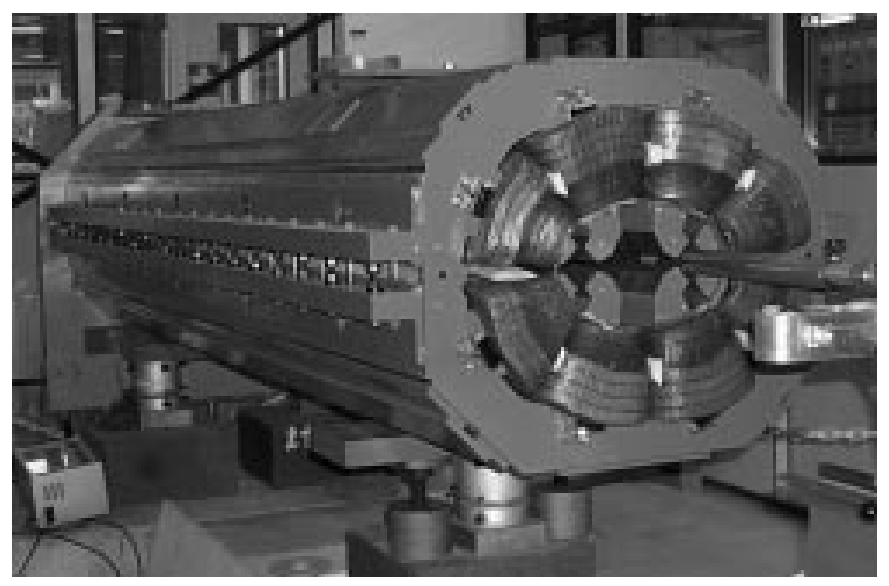

Fig. 2. MQW001 showing the nonconnection end. 

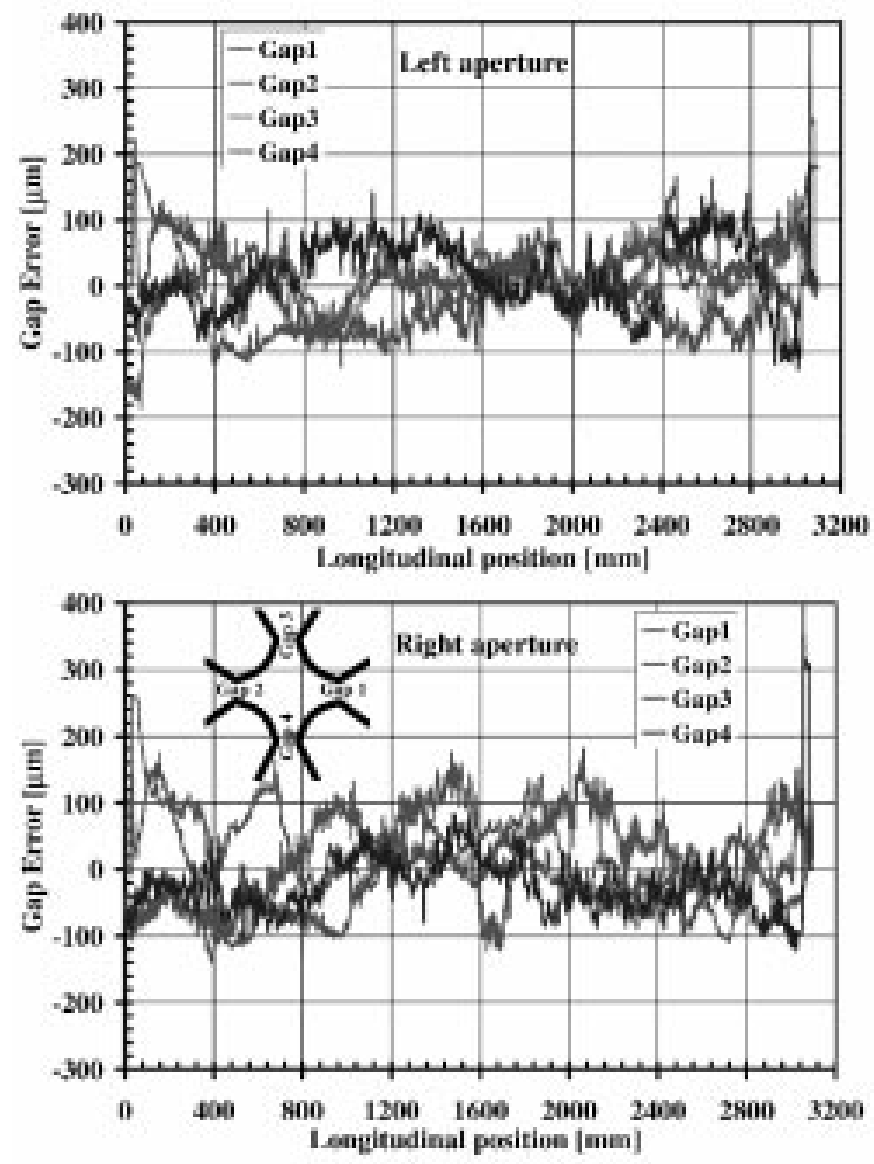

Fig. 3. Measured pole distance errors on MQW001 as function of the longitudinal position in the apertures. $L=0$ is at the nonconnection end, left/right as seen from the connection end of the magnet.

TABLE II

PDMD MEASUREMENT RESULTS SUMMARY

\begin{tabular}{|c|c|c|c|}
\hline Cristerion type & Limit & Left Apert. & Right Apert. \\
\hline & (\%) & (\%) & (5) \\
\hline $\mid$ Seap $<<0.1 \mathrm{~mm}$ & 390 & 96 & 91 \\
\hline $0.1 \mathrm{~mm}<|\Delta \mathrm{pap}|<0.15 \mathrm{~mm}$ & $<10$ & 4 & 9 \\
\hline Agap $\mid>0.15 \mathrm{~mm}$ & 0 & 0 & 0 \\
\hline 5 yoas $<0.3 \mathrm{~mm}$ & 390 & 97 & 95 \\
\hline $0.3 \mathrm{mim}<2 \mathrm{sgap}<0.45 \mathrm{~mm}$ & $<10$ & 3 & 5 \\
\hline Inap $>0.45 \mathrm{~mm}$ & 0 & 0 & 0 \\
\hline
\end{tabular}

pole. The following design changes were made with respect to the prototype:

- Full-length steel dowel pins in slots were added to the mating faces between the Type 2 stacks. The pins guarantee the precision in the relative positioning of the half magnets.

- A $100 \mu \mathrm{m}$ nominal slit was added between the mating faces of the Type 1 and Type2 stacks to absorb punching and stacking errors. At assembly this slit is filled with epoxy resin.

- Extra rows of bolts were added to assemble the two halfmagnets together. The exterior rows have push bolts; the inner rows have pull bolts. With the two types of bolts, the half magnets can be held together and the pole gaps can be adjusted.
- The option to bolt the Type1 and Type2 stacks together was added. The pre-series magnet was welded.

- The coils were redesigned to greatly reduce the number of brazed joints and potential leaks.

- The current and water connections were completely re-designed to be rapidly serviceable in a high radiation environment.

To implement these changes and to overcome the construction problems experienced with the prototype, the following tooling modifications were made:

- New punching die (pre-punch and final die).

- Reinforced and separate stacking tables for Type1 and Type2 stacks.

- Separate half-magnet assembly table with the primary reference datum in the pole gap space.

- New coil winding machine.

During construction, in addition to the usual checks, the following special measurements and verifications were made.

- The contacts between the poles and the stacking datums were verified on the half-magnet table by measuring the outer size of the half-magnets. A special measurement bridge was used.

- At final assembly, a pole distance measurement device (PDMD) [2] was used to measure the four distances between the poles in each beam aperture.

\section{Results of Mechanical Measurements}

The PDMD measures the deviation of the distances of the flat parts of the poles from the nominal value $(15 \mathrm{~mm})$ at $1 \mathrm{~mm}$ intervals along an aperture. The results of these measurements are shown in Fig. 3. The acceptance criteria for the magnet and the measured values are summarized in Table II.

Two types of criteria were applied, one on the absolute value of the pole distance deviation and one on the sum of the absolute values of the pole distance deviations at one longitudinal position. The measurements at the entry and exit of the aperture are discarded, as the device is not well guided there. Table II shows that the pole distances are within the specified tolerance [3].

\section{Results of Magnetic Measurements}

The magnetic field of the pre-series magnet was measured at CERN using a rotating coil mole [4]. The mole has an outer diameter of $41 \mathrm{~mm}$ and an effective length of $750 \mathrm{~mm}$. It is positioned in the apertures with the help of a support tube. The mole contains three harmonic coils. The measured field is represented as multipoles. The definition of the multipoles is given in reference [5]. The coefficients $b_{n}$ and $a_{n}$ represent respectively the normal and skew relative field errors at the reference radius where. They are dimensionless and are given in units of $10^{-4}$. The normal quadrupole is $b_{2}$.

The measured dipole component depends on how well the mole was centered in the aperture. Mole measurements were done at five longitudinal positions and added together to obtain the integrated field of the magnet including the end fields. The measurements were analyzed to obtain the multipole decomposition of the field. Measurements at the longitudinal center of 

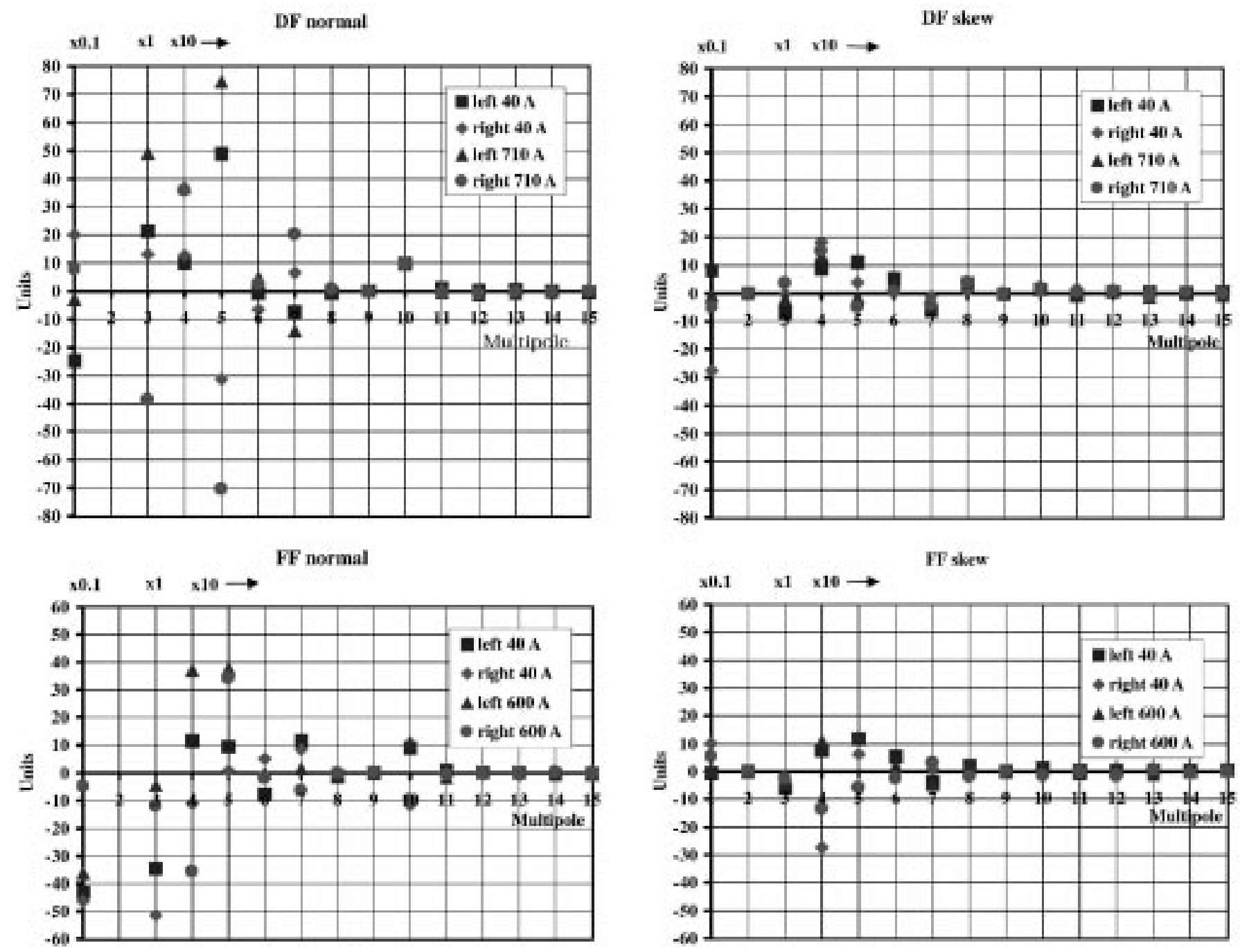

Fig. 4. Results of the magnetic measurements with the rotating coil mole on the pre-series magnet. The mole is introduced from the nonconnection end. Left/right is defined from the connection end. The top plots are for DF mode; the bottom plots are for FF mode. The data in the plots is scaled, for $n=1$ (dipole) the data is multiplied by 0.1 , for $n=4$ (octupole) and higher the data is multiplied by 10 . The quadrupole component $(n=2)$ is by definition $10^{4}$.

the magnet were used to obtain the field dependence on the excitation current.

\section{A. Polarity Issues}

The coils for the two apertures can be powered in two ways: such that the two counter-rotating LHC beams see a Defocusing-Focusing magnet (DF) or that the two beams see a Focusing-Focusing magnet (FF). Initially the magnet was designed to be powered in the DF mode only. The measurements were made with the mole entering from the nonconnection end, hence, in the DF mode, the mole will see both apertures as defocusing quadrupoles [6].

Multipole calculations were made on finite element models of an ideal magnet and one with mechanical errors. These calculations show a multipole sign difference of $(-1)^{n}$ between the two apertures, when the apertures are seen from the same end of the magnet. This effect is also seen in the measurements (Fig. 4).

\section{B. Multipoles of the Integrated Field}

In Fig. 4 the results can be found for all the measurements in DF and FF mode. A correction algorithm renormalizing $b_{9}$ and $a_{2}$ was applied. The dipole component has a large measurement error of 240 units due to the mole positioning uncertainty of 0.4 $\mathrm{mm}$. The sextupole component has an error of a few units, the higher order multipoles have measurement errors of one unit or less. In Fig. 5 the current dependence of the gradient is plotted. In the range between $100 \mathrm{~A}$ and $500 \mathrm{~A}$ the magnet is linear. Below 100 A the nonlinearity is caused by a lower steel permeability.

Above 500 A steel saturation sets in. We conclude that the magnet is usable in the range needed for the LHC (40 A Injection level, 710 A Nominal physics level, 810 A Ultimate physics level).

Mechanical errors and steel imperfections could cause the field distribution to vary along the length of the yoke. In Fig. 6 measurements of the five mole positions are shown for the DF case. The effects at the magnet ends (mole positions 1 and 5) are clearly visible for $b_{4}$ and $b_{6}$. 


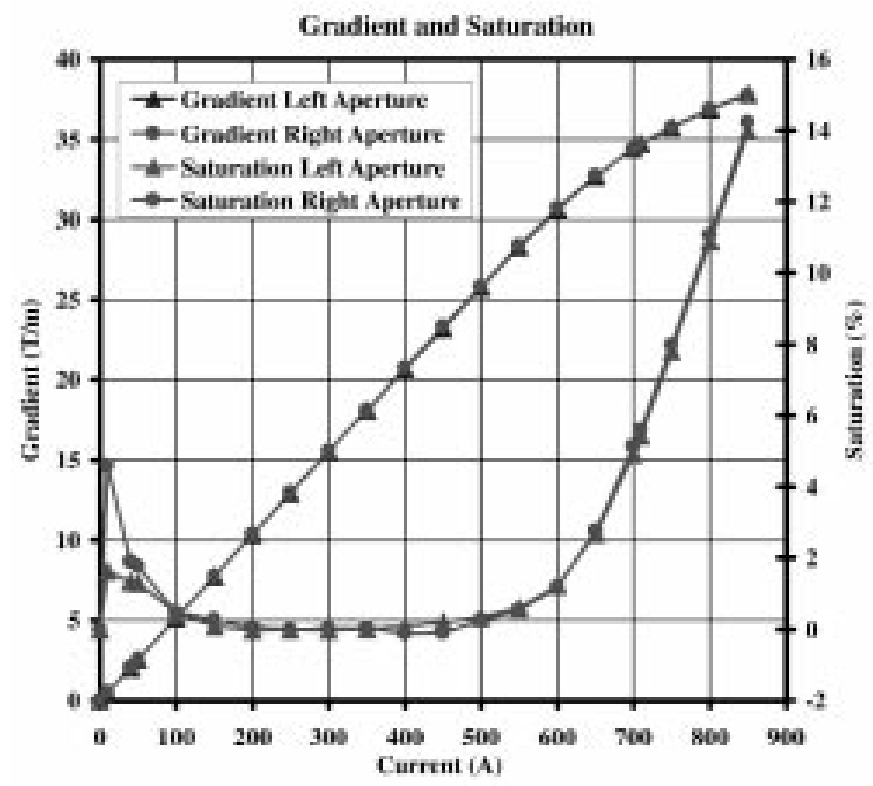

Fig. 5. Gradient $G$ for the center mole position and the saturation of this gradient as function of the excitation current.

\section{Comparison With Ideal Magnet}

Fig. 7 shows the results of a finite element calculation of the multipole components for the DF case for an ideal magnet (a homogeneous yoke and no mechanical errors). Comparing with the measurements, one can see that the imperfections generate mainly normal dipole and sextupole components. The $b_{10}$ of 0.8 units, as predicted by field calculations, is seen on the magnet. Compared to the prototype magnet, the MQW001 is much improved [2]. The $b_{3}$ and $b_{4}$ and the skew multipole components are smaller and the field varies less along the length of the yoke.

\section{FIELD CORRECTIONS TO BE APPLIED}

Preliminary results from beam dynamics calculations indicate that a major part of the sextupole components and of $b_{10}$ should be shimmed away or compensated. Measurements with two small shims per pole on the nonconnection end show a reduction of $b_{10}$ to 0.5 units. Further shimming possibilities, i.e., for the sextupole, are being studied.

\section{CONCLUSION}

Because of the narrow distance between the two apertures and the small aperture diameter, unprecedented mechanical tolerances in resistive quadrupole fabrication and assembly had to be realized in order to achieve the results for MQW001. We are now confident that we can produce magnets of sufficient quality. Consequently series production has started this summer.

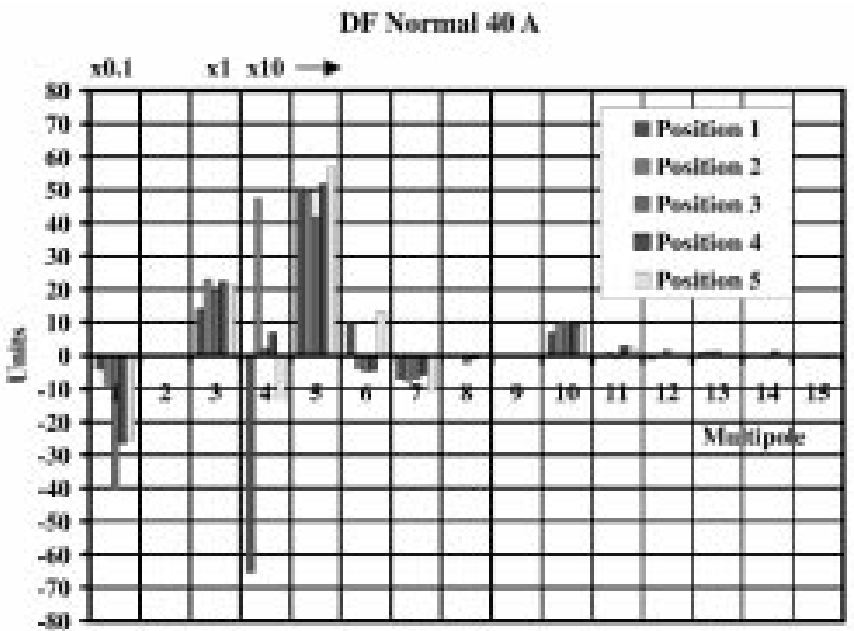

Fig. 6. Longitudinal distribution of the field. The measurements of the five mole positions for the DF case.

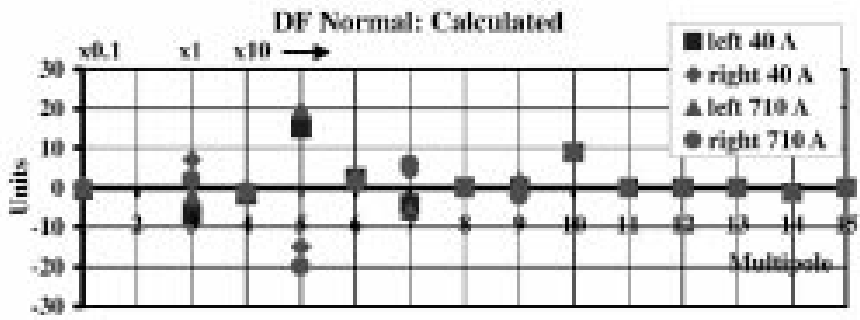

Fig. 7. Multipoles from a finite element calculation of an ideal magnet (a homogeneous yoke and no mechanical errors).

A second magnet was completed in August. The full production speed of two magnets per month should be achieved in October.

\section{ACKNOWLEDGMENT}

The authors would like to thank A. Astbury, E. Blackmore, L. Evans, and W. Kalbreier for their continuing and patient support of the MQW project.

\section{REFERENCES}

[1] R. Ostijic, presented at the Conf. MT-17.

[2] G. S. Clark, O. Hans, G. de Rijk, and M. Racine, "A twin aperture resistive quadrupole for the LHC," IEEE Trans. Appl. Superconduct., vol. 10, pp. 147-149, Mar. 2000.

[3] G. S. Clark, "Technical specification for the series production of the twin aperture quadrupoles for the LHC,", LHC-MQW-CI-0003.

[4] J. Billan, et al., "Ambient temperature field measuring system for LHC superconducting dipoles,", Tampere, Finland, LHC Note 332, MT-14, June, 11-16 1995.

[5] R. Wolf, "Field error naming conventions for LHC magnets, engineering specification,", LHC-MMS/ES/01, LHC-M-ES-0001.00 rev. 1.1.

[6] P. Proudlock, "LHC magnet polarities, engineering specification,", LHC-DC-ES-0001.00 rev.1.1. 